\title{
Reconstrução Nasal: análise de 253 casos realizados no Instituto Nacional de Câncer*
}

\author{
Nasal Reconstruction at the Brazilian National Cancer Institute: \\ experience in 253 cases
}

Marcus Vinicius Ponte de Souza Filho,' Rudolf Nunes Kobig, 'Patrícia Breder de Barros, 'Márcio José Abreu Dibe ${ }^{2} \mathrm{e}$

Paulo Roberto Albuquerque Leal ${ }^{3}$

\section{Resumo}

Foram analisados 253 casos de reconstrução nasal, 80,63\% devido a carcinoma basocelular e $7,51 \%$ devido a carcinoma espinocelular. As principais áreas comprometidas foram: dorso $(41,50 \%)$, asa (30,43\%), ponta (24,90\%) eparedelateral (12,25\%). Excluindo-se o avançamento direto simples, o principal procedimento utilizado na reconstrução do dorso foi o retal ho dorso-glabelar (17,14\%); da asa nasal, o retalho nasogeniano $(55,84 \%)$; da ponta, o retalho bilobado $(20,63 \%)$ e da parede lateral, o retalho nasogeniano $(58,06 \%)$. Para os casos de reconstrução total de nariz $(2,77 \%)$ o procedimento mais utilizado foi o retalho de Converse $(71,42 \%)$.

Palavras-chaves: neoplasias nasais; carcinoma; nariz; reconstrução; retalhos cirúrgicos.

\section{Abstract}

In the present study, the authors analyse 253 cases of nose reconstruction, $80.63 \%$ due to basal cell carcinoma and $7.51 \%$ due to squamous cell carcinoma. The main nasal subunits involved are distributed as follows: dorsum $(41.50 \%)$, alae (30.43), tip (24.90\%) and sidewall (12.25\%). Excluding the simple direct advancement, the main surgical technique employed for reconstruction of the dorsum wasthe dorsum-glabelar flap (17.14\% ), of thealaewasthenasolabial flap (55.84\%), of the tip was the bilobed flap (20.63\%) and of the sidewall was the nasolabial flap (58.06\%). In the cases of total nose reconstruction $(2.77 \%)$ the Converse scalping flap was the principal procedure used $(71.42 \%)$.

Key words. nose neoplasms, carcinoma; nose; reconstruction; surgical flaps.

\footnotetext{
*Trabalho de Conclusão de Curso apresentado ao Instituto N acional de Câncer para obtenção de T ítulo de Residência em Cirurgia Plástica.

${ }^{1} M$ édico Residente. Serviço de Cirurgia Plástica Reparadora e M icrocirurgia, Instituto N acional de Câncer (IN CA). ${ }^{2} \mathrm{M}$ édico Assistente. Serviço de Cirurgia Plástica Reparadora e M icrocirurgia, Instituto N acional de Câncer (IN CA). ${ }^{3}$ Chefe do Serviço de Cirurgia Plástica Reparadora e M icrocirurgia, Instituto N acional de Câncer (IN CA). Enviar correspondência para P.R.A.L. IN CA, Serviço de Cirurgia Plástica Reparadora e M icrocirurgia, 80 andar, Praça da Cruz Vermelha 23, Centro, 22230-130 Rio de Janeiro, RJ - Brasil.

Recebido em janeiro de 2002.
} 


\section{INTRODUÇÃO}

A reconstrução nasal é a mais antiga das operações plásticas. Trinta séculos antes da era C ristã já era praticada por sacerdotes, como aparece no papiro de Edwin Smith. Em 600 a.C., Sushruta descreve no livro sagrado dos $\mathrm{H}$ indus a reconstrução do nariz com retal ho frontal egeniano. $\mathrm{N}$ o século XVI, Tagliacozzi publica um tratado sobre o uso do retalho pediculado braquial. ${ }^{1} 0$ retalho

frontal foi primeiramente usado no mundo ocidental por Joseph Carpue na Inglaterra, quando em 1816 publicou dois relatos de casos. N este século Gilies, Converse e M illard foram pioneiros nesta modalidade de cirurgia. ${ }^{2-4}$

Com a melhoria das ações preventivas de saúde e conseqüente diagnóstico mais precoce, as grandes ressecções nasais estão diminuindo cada vez mais a sua freqüência, tornando a reconstrução total de nariz um acontecimento quase que particular de hospitais especializados em oncologia. Entretanto os casos de reconstruções parciais podem muitas vezes ser realizados através de procedimentos simples e criativos.

O s tumores, em especial o carcinoma basocelular e o carcinoma epidermóide, constituem a principal indicação clínicocirúrgica das reconstruções nasais. ${ }^{5} 0$ utras indicações são: anomalias, radiodermite crônica, traumatismos e infecções. ${ }^{6}$

0 objetivo deste trabalho foi realizar uma análise retrospectiva de 253 pacientes submetidos à reconstrução nasal no Instituto $\mathrm{N}$ acional de $\mathrm{C}$ âncer, com avaliação das principais técnicas cirúrgicas abordadas para reconstrução das diversas regiões nasais acometidas.

\section{PACIENTES E MÉTODOS}

D urante o período de 10 de fevereiro de 1999 a 31 de janeiro de 2000, 253 pacientes foram submetidos à reconstrução parcial ou total de nariz no Instituto $\mathrm{N}$ acional de Câncer (INCA).

A avaliação dos casos foi realizada retrospectivamente através da análise de prontuários, sendo os parâmetros estudados os seguintes: a) sexo; b) idade; c) cor; d) indicação clínico-cirúrgica para reconstrução nasal; e) tamanho do defeito; f) subunidade(s) estética(s) nasal(is) a ser(em) reconstruída(s) - dorso, parede lateral, ponta, asa nasal e/ou columela; ${ }^{7}$ g) tipo de técnica cirúrgica empregada na reconstrução - avançamento direto simples, retalho dorso-glabelar, retalho bilobado, retalho de dorso nasal, retalho médio-frontal, retalho nasogeniano, retalho labial superior, retalho de Converse, autoenxertia cutânea e outros; e h) tipo de procedimento anestésico utilizado para realização da reconstrução nasal.

Os diversos parâmetros foram analisados de duas formas: uma análise geral e uma análise em função da subunidade nasal comprometida.

\section{RESULTADOS}

Resultados gerais

D os 253 pacientes estudados $94,07 \%$ eram brancos e $5,93 \%$ eram pardos (Figura 1), $50,59 \%$ do sexo masculino e $49,41 \%$ do sexo feminino (Figura 2). A média de idade dos pacientes foi de 66 anos, sendo a principal indicação clínico-cirúrgica para reconstrução nasal o carcinoma basocelular, responsável por $80,63 \%$ dos casos, seguido pelo carcinoma epidermóide (7,51\%) (Figura $3)$. 0 restante dos defeitos surgiu pósressecção cirúrgica de carcinoma cilindromatoso, carcinoma tricoblástico, cisto-hidroadenoma papilífero, carcinoma sebáceo, foliculite crônica granulomatosa e fibrosante, ceratose actínica, ceratose seborréica e outros. Em 2,77\% dos casos foi realizada reconstrução total do nariz e no restante dos casos reconstruções parciais ou subtotais. A principal subunidade estética nasal atingida foi o dorso (41,50\%), seguido pela asa nasal $(30,43 \%)$, ponta nasal $(24,90 \%)$, parede lateral nasal $(12,25 \%)$ e columela $(1,19 \%$ ) (Figura 4). Em $11.86 \%$ dos casos houve acometimento de mais de uma subunidade estética. A média do tamanho dos defeitos criados pós-ressecção foi de $2,41 \mathrm{~cm}$ $x 1,76 \mathrm{~cm}$, sendo o maior defeito de $7,50 \mathrm{~cm}$ $x 7,00 \mathrm{~cm}$. 0 procedimento de reconstrução 
nasal foi realizado em $71,94 \%$ dos casos sob anestesia local, $20.16 \%$ sob anestesia local com sedação, e 7,90\% sob anestesia geral inalatória (Figura 5).

Figura 1. Distribuição dos Pacientes de Acordo com a Cor.

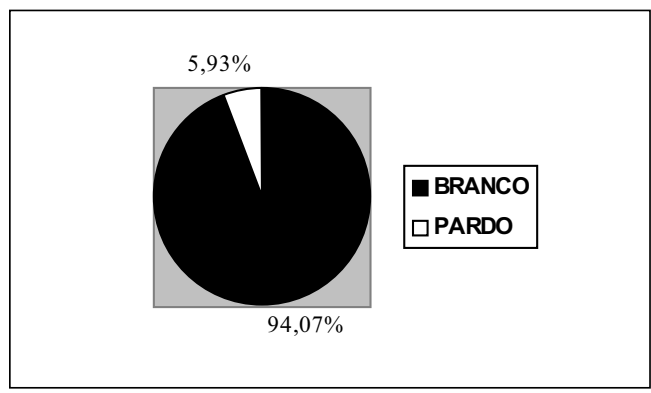

Figura 2. Distribuição dos Pacientes de Acordo com o Sexo.

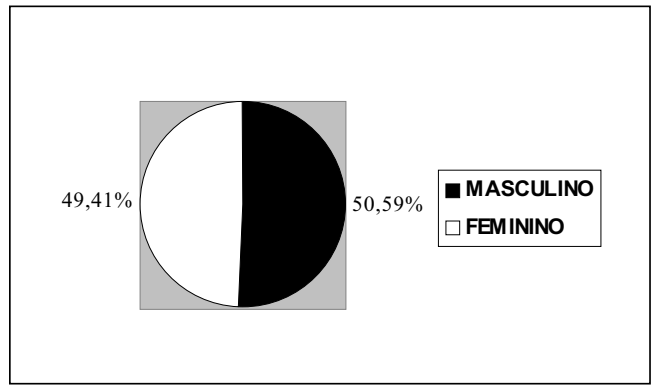

Figura 3. Distribuição dos Pacientes de Acordo com a Indicação Clínico-Cirúrgica para Reconstrução Nasal.

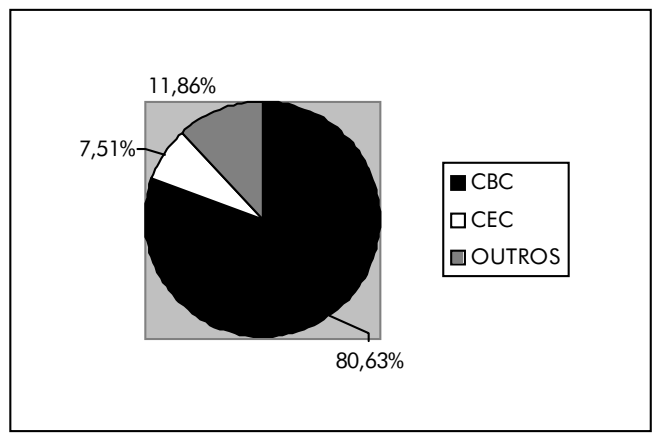

Figura 4. Localização dos Defeitos por Subunidades Estéticas Nasais.

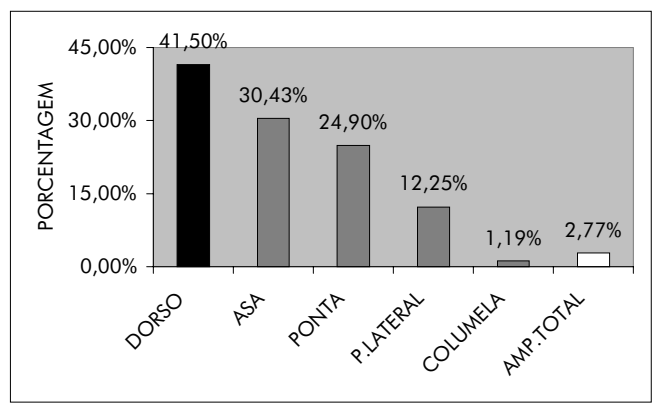

Figura 5. Distribução dos Pacientes de Acordo com o Tipo de Procedimento Anestésico Utilizado.

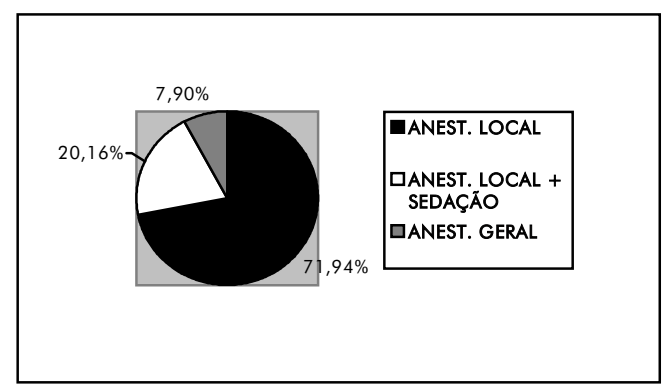

Resultados em função da subunidade estética nasal comprometida

\section{Dorso nasal}

Em 105 casos estudados houve comprometimento do dorso nasal, sendo a média do tamanho dos defeitos neste grupo de $2,59 \mathrm{~cm} \times 1,85 \mathrm{~cm}$.

As principais técnicas cirúrgicas empregadas na reconstrução destes defeitos foram: a) avançamento direto simples $(43,81 \%) ;$ b) retalho dorso-glabelar $(17,14 \%)$; c) retalho médio-frontal $(16,19 \%)$; e d) autoenxertia cutânea $(9,52 \%)$ (Figura 6A).

$\mathrm{N}$ os casos de auto-enxertia cutânea as principais áreas doadoras utilizadas foram: a) região supra-clavicular (50\%); b) região infra-clavicular (20\%); c) região sub-mentoniana (20\%); e d) região retro-auricular (10\%).

Figura 6A. Procedimentos Cirúrgicos Realizados para Reconstrução de Defeitos Acometendo o Dorso Nasal.

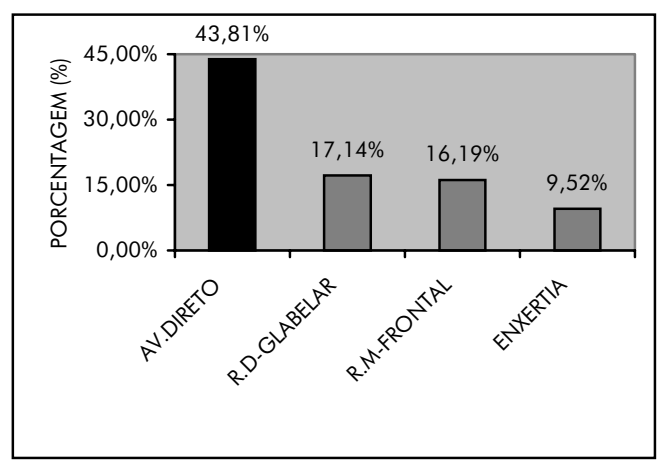

A média do tamanho dos defeitos no grupo em que foi realizado avançamento direto simples foi de $1.73 \mathrm{~cm} \times 1.11 \mathrm{~cm}$, enquanto esta média foi de $2.44 \mathrm{~cm} \times 1.81 \mathrm{~cm}$ para 0 grupo em que se utilizou o retalho dorso-glabelar, $4.46 \mathrm{~cm} \times 3.25 \mathrm{~cm}$ no grupo em que se utilizou o retalho médio-frontal, $e$ 
$4.49 \mathrm{~cm} \times 3.44 \mathrm{~cm}$ nos casos em que foi realizada auto-enxertia cutânea (Figura 6B).

Figura 6B. Média de Tamanho dos Defeitos de Dorso Nasal das Duas Maiores Medidas Associada ao Tipo de Procedimento Cirúrgico Utilizado.

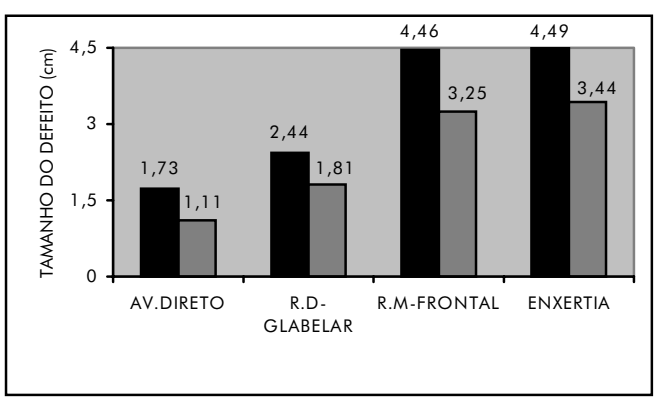

Parede lateral nasal

E m 31 casos estudados houve comprometimento da parede lateral nasal, sendo a média do tamanho dos defeitos neste grupo de $2,26 \mathrm{~cm} \times 1,75 \mathrm{~cm}$.

As principais técnicas cirúrgicas empregadas na reconstrução destes defeitos foram: a) retalho nasogeniano $(58,06 \%)$; b) avançamento direto simples $(29,03 \%)$; e c) retalho médio-frontal $(9,68 \%)$ (Figura $7 \mathrm{~A})$.

A média do tamanho dos defeitos no grupo em que foi realizado avançamento direto simples foi de $1,41 \mathrm{~cm} \times 0,81 \mathrm{~cm}$, enquanto esta média foi de $2,34 \mathrm{~cm} \times 1,91 \mathrm{~cm}$ para 0 grupo em que se utilizou o retalho nasogeniano, e $4,50 \mathrm{~cm} \times 3,57 \mathrm{~cm}$ nos casos em que foi realizado 0 retalho médio-frontal (Figura 7b).

Figura 7A. Procedimentos Cirúrgicos Realizados para Reconstrução de Defeitos Acometendo a Parede Lateral Nasal.

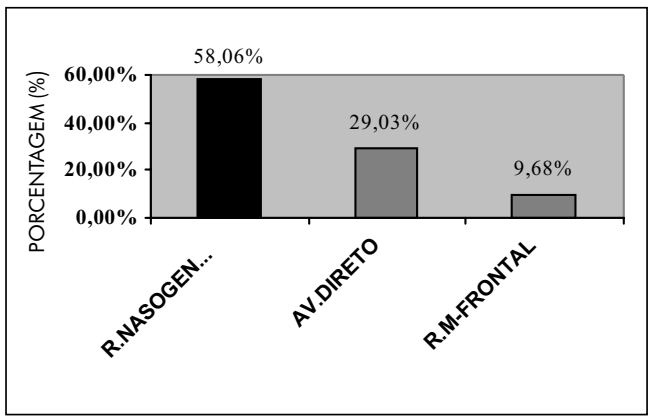

Ponta nasal

Em 63 casos estudados houve comprometimento da ponta nasal, sendo a média do tamanho dos defeitos neste grupo de $2,43 \mathrm{~cm} \times 1,75 \mathrm{~cm}$.

Figura 7B. Média de Tamanho dos Defeitos de Parede Lateral Nasal das Duas Maiores Medidas Associada ao Tipo de Procedimento Cirúrgico Utilizado.

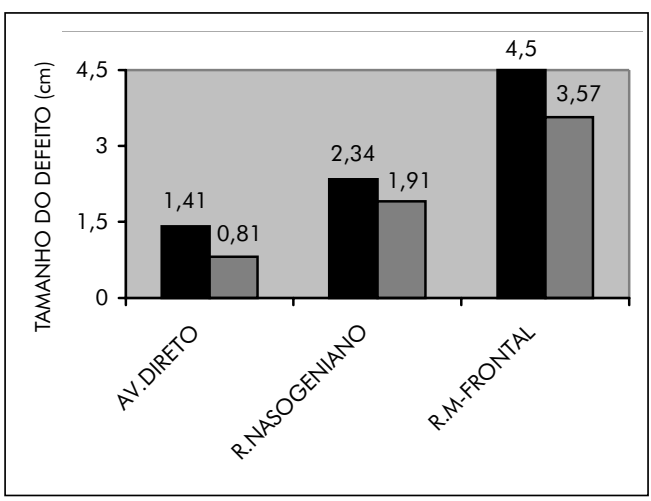

As principais técnicas cirúrgicas empregadas na reconstrução destes defeitos foram: a) avançamento direto simples $(26,98 \%)$; b) retalho bilobado $(20,63 \%)$; c) retalho do dorso nasal $(17,46 \%)$; e d) retalho médio-frontal (17.46\%) (Figura $8 \mathrm{~A})$.

Figura 8A. Procedimentos Cirúrgicos Realizados para Reconstrução de Defeitos Acometendo a Ponta Nasal.

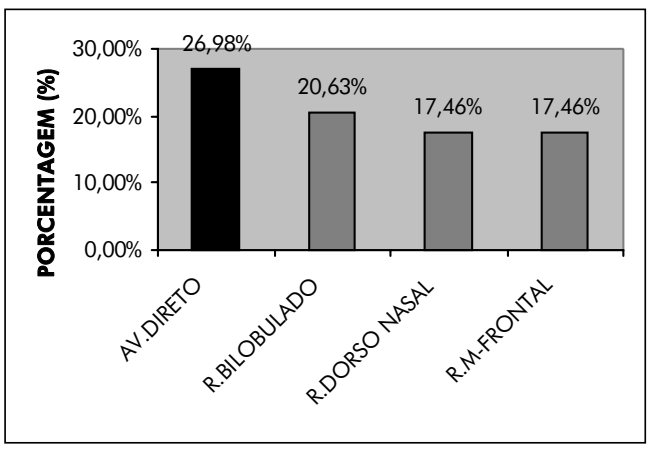

A média do tamanho dos defeitos no grupo em que foi realizado avançamento direto simples foi de $1,59 \mathrm{~cm} \times 1,05 \mathrm{~cm}$, enquanto esta média foi de $1,69 \mathrm{~cm} \times 1,18 \mathrm{~cm}$ para o grupo em que se utilizou o retalho bilobado, $2,04 \mathrm{~cm} \times 1,46 \mathrm{~cm}$ no grupo em que se utilizou o retalho do dorso nasal, e $4.01 \mathrm{~cm}$ $x 3.05 \mathrm{~cm}$ nos casos em que foi realizado retalho médio-frontal (Figura $8 \mathrm{~B}$ ).

Asa nasal

Em 77 casos estudados houve comprometimento da asa nasal, sendo a média do tamanho dos defeitos neste grupo de $2,46 \mathrm{~cm} \times 1,85 \mathrm{~cm}$. 
Figura 8B. Média de Tamanho dos Defeitos de Ponta Nasal das Duas Maiores Medidas Associada ao Tipo de Procedimento Cirúrgico Utilizado.

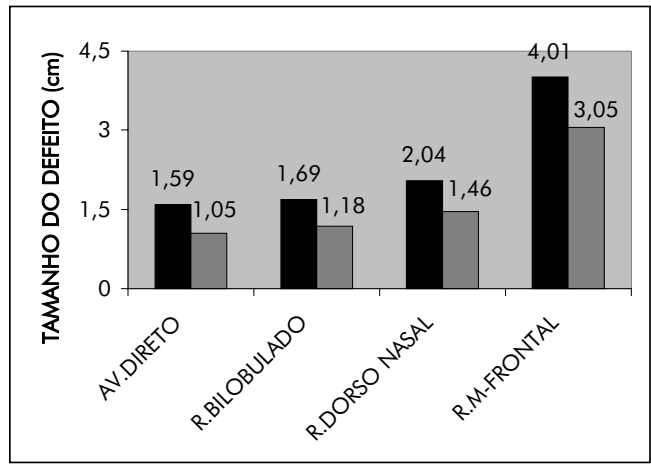

As principais técnicas cirúrgicas empregadas na reconstrução destes defeitos foram: a) retalho nasogeniano $(55,84 \%)$; b) avançamento direto simples $(20,78 \%)$; e c) retalho médio-frontal (16,88\%) (Figura 9A).

A média do tamanho dos defeitos no grupo em que foi realizado avançamento direto simples foi de $1,19 \mathrm{~cm} \times 0,77 \mathrm{~cm}$, enquanto esta média foi de $2,59 \mathrm{~cm} \times 2,02 \mathrm{~cm}$ para 0 grupo em que se utilizou 0 retalho nasogeniano, e $4,64 \mathrm{~cm} \times 3,38 \mathrm{~cm}$ nos casos em que foi realizado o retalho médio-frontal (Figura 9B).

Figura 9A. Procedimentos Cirúrgicos Realizados para Reconstrução de Defeitos Acometendo a Asa Nasal.

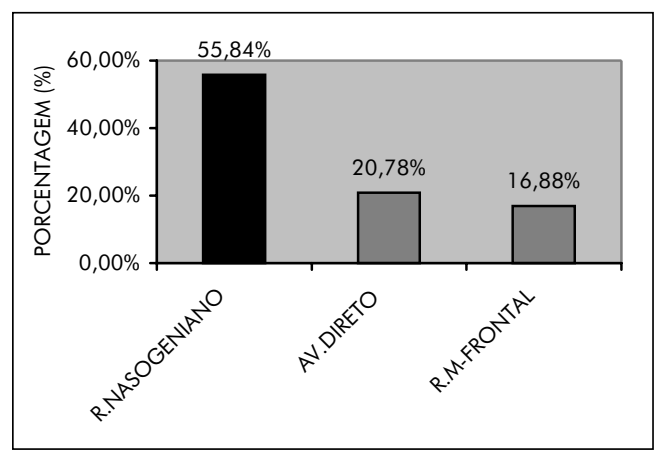

Columela

Em três casos estudados houve comprometimento da columela (excluindo-se os casos em que foi realizada amputação total do nariz), sendo a média do tamanho dos defeitos neste grupo de $2,37 \mathrm{~cm} \times 1,77 \mathrm{~cm}$.

As principais técnicas cirúrgicas empregadas na reconstrução destes defeitos foram: a) retalho labial superior em rotação
(66,67\%); e b) avançamento direto simples (33,33\%) (Figura 10A).

Figura 9B. Média de Tamanho dos Defeitos de Asa Nasal das Duas Maiores Medidas Associada ao Tipo de Procedimento Cirúrgico Utilizado.

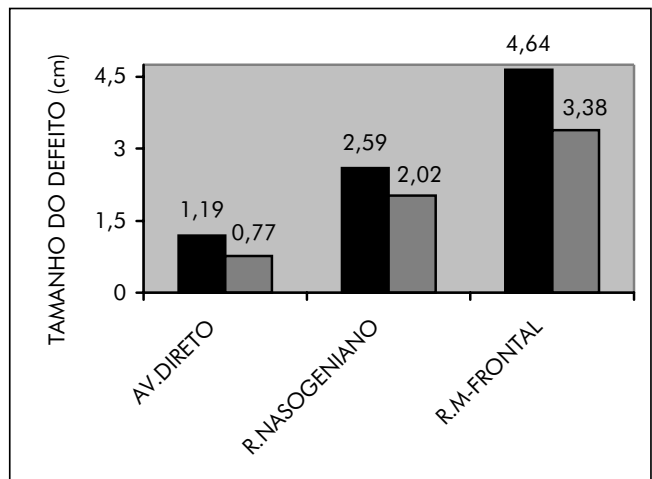

A média do tamanho dos defeitos no grupo em que foi realizado avançamento direto simples foi de $1,50 \mathrm{~cm} \times 1,00 \mathrm{~cm}$, enquanto esta média foi de $2,80 \mathrm{~cm} \times 2,15 \mathrm{~cm}$ nos casos em que foi realizado o retalho labial superior em rotação (Figura 10B).

Figura 10A. Procedimentos Cirúrgicos Realizados para Reconstrução de Defeitos Acometendo a Columela Nasal.

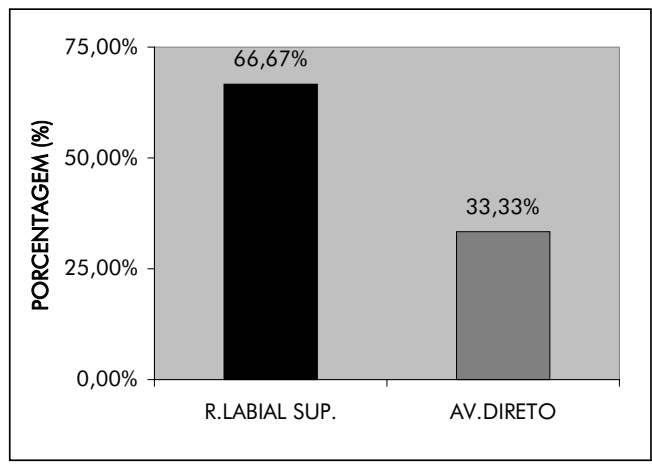

Amputação total do nariz

A indicação clínico-cirúrgica para reconstrução nasal total nestes casos foi em $71,43 \%$ devido à presença de carcinoma epidermóide, enquanto em $28,57 \%$ foi devido a carcinoma basocelular. A média do defeito reconstruído foi de $6,53 \mathrm{~cm} \times 5,50 \mathrm{~cm}$.

As principais técnicas utilizadas para reconstrução nasal total foram: a) retalho de Converse $(71.42 \%)$; b) retalho médio-frontal (14.29\%); e c) retalho retro-auricular de pedículo contra-lateral - retalho de Galvão 
(14.29\%) (Figura 11A).

Figura 10B. Média de Tamanho dos Defeitos de Columela Nasal das Duas Maiores Medidas Associada ao Tipo de Procedimento Cirúrgico Utilizado.

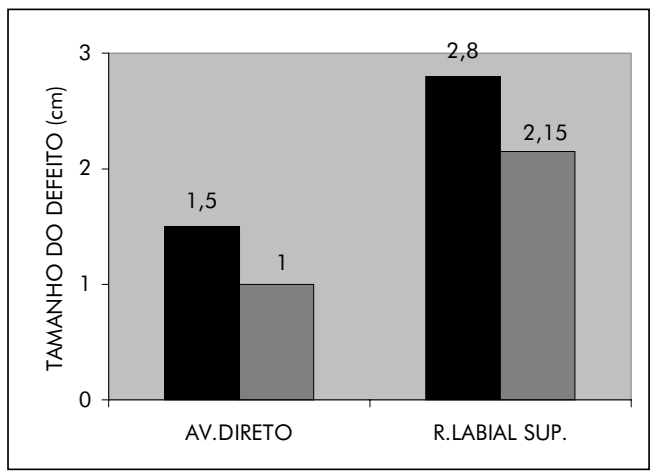

Figura 11A. Procedimentos Cirúrgicos Realizados para Reconstrução Total de Nariz.

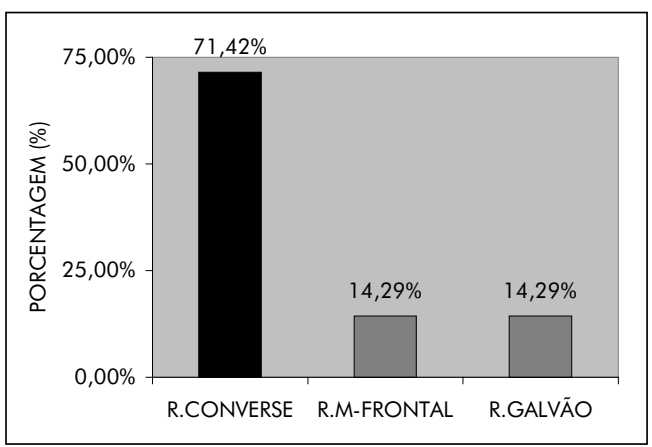

A média do tamanho dos defeitos em que foi realizado 0 retalho de Converse foi de $6,80 \mathrm{~cm} \times 5,70 \mathrm{~cm}$, enquanto no caso em que se realizou o retalho médio-frontal este valor foi de $4,70 \mathrm{~cm} \times 4,00 \mathrm{~cm}$, e no caso em que se empregou o retal ho retro-auricular de pedículo contra-lateral este valor foi de $7,50 \mathrm{~cm} x$ $7,00 \mathrm{~cm}$ (Figura 11B).

Figura 11B. Média de Tamanho dos Defeitos Nasais das Duas Maiores Medidas Associada ao Tipo de Procedimento Cirúrgico Utilizado para Reconstrução Total de Nariz.

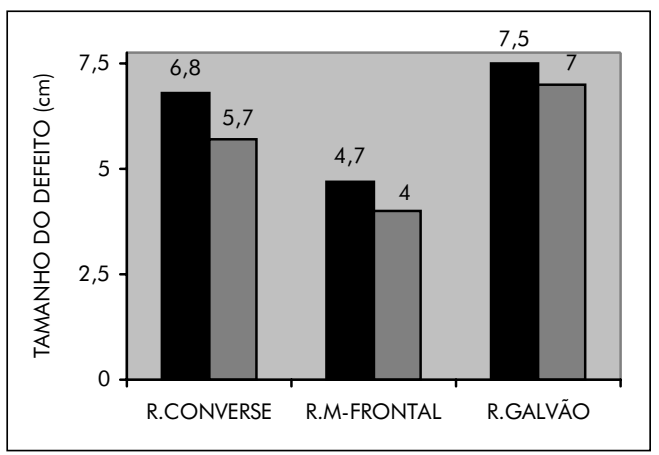

\section{DISCUSSÃO E CONCLUSÃO}

0 carcinoma basocelular foi o principal responsável pelos defeitos nasais em que se realizaram reconstruções parciais ou subtotais de nariz, enquanto o carcinoma epidermóide foi o principal responsável nos casos de reconstrução nasal total.

0 câncer de pele que não o melanoma cutâneo, mesmo apresentando um difícil estudo epidemiológico e com estimativa de altos índices de subnotificação, é o tipo de câncer notificado mais freqüente entre os homens brasileiros e o segundo entre as mulheres. ${ }^{8}$ A proximadamente $93 \%$ dos carcinomas basocelulares ocorrem na cabeça e pescoço, e destes, $25 \%$ aparecem na pirâmide nasal, de forma que este indubtavelmente é o câncer mais comum da cabeça e pescoço. ${ }^{9,10}$

Em nosso estudo $80,63 \%$ das lesões encontradas no nariz foram carcinomas basocelulares, o que está de acordo com os estudos de Fava eC arvalho ${ }^{11}$ que encontraram uma incidência de $80 \%$ em 87 casos estudados.

Em nossa experiência, a distribuição topográfica das lesões tumorais nasais com indicação para reconstrução nasal apresentou uma maior incidência na região do dorso nasal $(41,50 \%)$, o que está de acordo com 0 trabalho de Soares; ${ }^{5}$ entretanto, em nosso estudo, houve uma maior incidência de lesões na ponta e na asa nasal, e uma menor incidência na parede lateral. Essa maior incidência de lesões na porção inferior da pirâmide nasal, possivelmente se deve ao fato de nosso serviço ser referência neste tipo de patologia, havendo, assim, encaminhamento de uma maior percentagem de casos complexos, o que freqüentemente envolve reconstruções do terço inferior do nariz. ${ }^{4}$

Em relação à subunidade estética nasal em que foi possível a reconstrução com avançamento direto simples, observou-se que o dorso nasal foi o local com maior incidência deste procedimento $(43,81 \%)$, sendo também - local com maior média de tamanho dos defeitos para realização desta técnica $(1,73 \mathrm{~cm}$ $x 1,11 \mathrm{~cm})$. Por outro lado, a asa nasal foi a subunidade estética com menor incidência deste procedimento $(20,78 \%)$ e com menor média de tamanho dos defeitos para realização 
desta técnica (1,19cm x 0,77cm) (Figura 12). Este fato ocorre devido ao relativo excesso de pele na região do dorso nasal e às características particulares da pele desta região que é fina, lisa e flexível, ao passo que a pele da subunidade estética da asa nasal é grossa, dura e pouco flexível. ${ }^{12}$

Figura 12. Média de Tamanho dos Defeitos das Duas Maiores Medidas para Reconstrução com Avançamento Direto Simples Associada à Subunidade Estética Nasal Acometida.

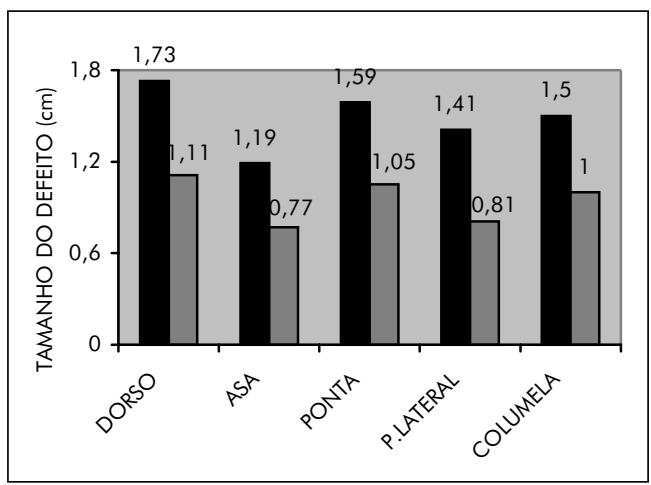

Excluindo-se 0 avançamento direto simples, observamos que o principal procedimento utilizado na reconstrução da subunidade do dorso foi o retalho dorsoglabelar $(17,14 \%)$, da parede lateral foi 0 retalho nasogeniano $(58,06 \%)$, da ponta foi o retalho bilobado $(20,63 \%)$, da asa nasal foi o retal ho nasogeniano $(55,84 \%)$ e da columela foi 0 retalho labial superior em rotação $(66,67 \%)$. Para os casos de reconstrução total de nariz o procedimento mais utilizado foi o retalho de Converse $(71,42 \%)$.

Com este trabalho pretendemos mostrar que as diversas subunidades estéticas nasais podem ser reconstruídas por uma multiplicidade de técnicas cirúrgicas, e cada reconstrução dependerá do local e do tamanho do defeito, da característica da pele e da forma do nariz do paciente, da familiaridade do cirurgião com determinados retalhos, bem como de sua criatividade no planejamento do ato operatório.

\section{REFERÊNCIAS BIBLIOGRÁFICAS}

1. González-U lloa M. The creation of aesthetic plastic surgery. N ew York: Springer-Verlag; 1985.

2. GilliesH D. Plastic surgery of the face. London: Oxford M edical; 1920.

3. Converse JM. Reconstruction of the nose by the scal ping flap technique. Surg Clin N orth Am 1959;39:335.

4. M illard DR Jr. Reconstructive rhinoplasty for thelower two-thirds of thenose. Plast Reconstr Surg 1976;57(6):722-8.

5. SoaresVR. Reconstrução denariz em neoplasias. Rev Bras M ed 1975;32(1):3-9.

6. Wintsch K. Rhinoplasty in leprosy. Plast Reconstr Surg 1968;42:208-10.

7. Burget GC, M enick FJ. The subunit principle in nasal reconstruction. Plast Reconstr Surg 1985;76:239.

8. M inistério da Saúde. Secretaria $N$ acional de Assistência à Saúde. Instituto $\mathrm{N}$ acional de Câncer (Brasil). Controle do câncer: uma proposta de integração ensino-serviço. 3a ed. Rio deJ aneiro: IN CA; 1999.

9. M arksR. An overview of skin cancer: incidence and causation. C ancer 1995;75:607-12.

10. $M$ arksR. Theepidemiology of non-melanoma skin cancer: who, why and what can we do about it. J D ermatol 1995;22:853-7.

11. FavaAS, Carvalho M B. Tumores malignos do nariz, cavidades nasais e seios paranasais. In: Brandão LG , FerrazAR. C irurgia de cabeçae pescoço. São Paulo: Roca; 1989.

12. G onzalez-U Iloa M . Restoration of thefacecovering by mean s of selected skin in regional aesthetic units. Br J Plast Surg 1956;9:212. 